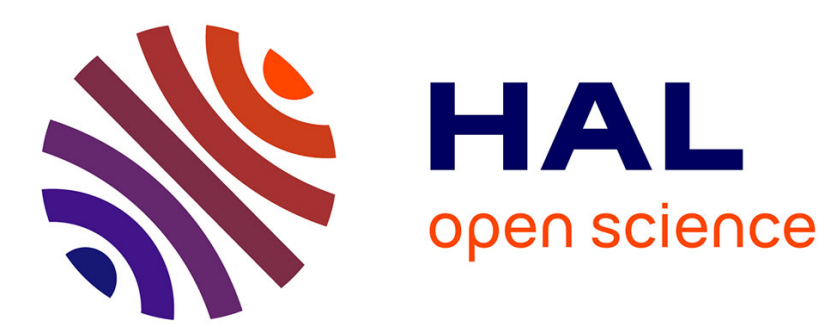

\title{
ACTIVITÉ THROMBOPLASTINIQUE DU LAIT. (Première note)
}

F. Brughera, P. Salvadori, F. Pellegrino

\section{To cite this version:}

F. Brughera, P. Salvadori, F. Pellegrino. ACTIVITÉ THROMBOPLASTINIQUE DU LAIT. (Première note). Le Lait, 1966, 46 (453_454), pp.159-168. hal-00928404

\section{HAL Id: hal-00928404 \\ https://hal.science/hal-00928404}

Submitted on 1 Jan 1966

HAL is a multi-disciplinary open access archive for the deposit and dissemination of scientific research documents, whether they are published or not. The documents may come from teaching and research institutions in France or abroad, or from public or private research centers.
L'archive ouverte pluridisciplinaire HAL, est destinée au dépôt et à la diffusion de documents scientifiques de niveau recherche, publiés ou non, émanant des établissements d'enseignement et de recherche français ou étrangers, des laboratoires publics ou privés. 


\title{
ACTIVITÉ THROMBOPLASTINIQUE DU LAIT
}

\author{
(Première note)
}

par

\author{
F. BRUGHERA, P. SALVADORI, F. PELLEGRINO \\ Istituto Provinciale Protezione \\ Assistenza Infanzia, Milano \\ (Dir. Gen. Prof. C. Torricelli) \\ Centro Sperimentale del Latte, Milano
}

\begin{abstract}
Avant-propos
L'expérimentation suivante trouve ses origines dans les tentatives que nous avons effectuées ces deux dernières années, d'influencer la coagulation du lait de vache cru avec des hémoanticoagulants.

Nous avons employé de l'héparine et du dicoumarol.

En résumant brièvement les résultats obtenus (qui seront publiés ensuite in extenso) nous pouvons affirmer que l'héparine influence la coagulation du lait de vache par la présure en la retardant progressivement, proportionnellement aux quantités d'héparine employées. Pour mettre en évidence les différents temps de coagulation, nous avons suivi la méthode normalement employée pour le titrage des présures. Les échantillons de lait employés $\left(100 \mathrm{~cm}^{3}\right)$ provenaient de la même masse et ont été additionnés avec des doses proportionnelles d'héparine à 5 p. 100 à raison de 0,$16 ; 0,32 ; 0,50 ; 1 ; 2$ et $5 \mathrm{~cm}^{3}$.

Les temps de coagulation variaient à partir d'un minimum de $2 \mathrm{mn} 48 \mathrm{~s}$ de l'échantillon témoin, jusqu'à $8 \mathrm{mn} 33$ s de l'échantillon contenant la dose maximum d'héparine.

Il y a lieu de remarquer que même les concentrations les plus faibles d'héparine empêchaient la séparation de sérum limpide, tandis que les concentrations les plus hautes limitaient le phénomène de la coagulation à l'apparition retardée de petits flocons caséeux, sans évolution ultérieure du coagulum.

Puisqu'on croyait possible une interférence de l'héparine sur l'action enzymatique de la présure, on a effectué des épreuves de coagulation chimique en ajoutant de $\mathrm{l}^{\prime} \mathrm{HCl}$ à peu près normal à $35^{\circ} \mathrm{C}$, suffisant pour atteindre le point isoélectrique de la caséine $(p \mathrm{H} 4,6)$.
\end{abstract}


A l'échantillon de lait de vache cru, venant de la même masse, on a ajouté de l'héparine à 5 p. 100 , les quantités échelonnées étant égales aux doses susmentionnées.

Dans ce second essai nous avons remarqué une interférence de l'héparine sur la structure du coagulum, qui se différenciait par une consistance réduite et une dispersion plus marquée à partir des concentrations les plus faibles d'héparine (fig. 1 et 2). Les mêmes expériences répétées en employant du dicoumarol comme substance anticoagulante éventuelle, ont donné des résultats négatifs.

Etant donnés les résultats des épreuves de coagulation sur le lait héparinisé et l'issue négative des épreuves après l'adjonction de dicoumarol, nous avons jugé opportun de faire des recherches, même si c'était seulement dans un sens qualitatif, dans le domaine de l'hémocoagulation, ou mieux d'établir si le lait ne serait pas capable d'exercer une action semblable à celle de quelque facteur hémocoagulant.

Il y a déjà d'autres auteurs dans ces dernières décennies, qui avaient effectué des recherches sur les analogies éventuelles entre le sang et le lait; nous citerons à ce sujet les travaux de Buruiana qui, en 1935 dénommait "fibrinogénase " [I] cette diastase du lait qui aurait la propriété de coaguler le fibrinogène à la température de $18-20^{\circ}$ C. Quelque temps après, Kopaczewski, dans sa monographie "Physico-chimie du lait» [2] dédiait un chapitre aux rapports biochimico-physiques existant entre le sang et le lait. Encore tout dernièrement d'autres savants ont réalisé des recherches thrombo-élastographiques sur le coagulum du lait, avec des résultats pas tout à fait déterminants [3-4]. Nos recherches préliminairement ont été effectuées sur les trois phases de coagulation, en employant du plasma de lait de vache comme facteur déterminant aux effets du mécanisme de coagulation de chaque phase séparée. (Par plasma du lait on entend la portion surnageante obtenue par la centrifugation du lait à $15000 \mathrm{t} / \mathrm{mn}$ pour 40 minutes.)

Nous tenons à souligner que le plasma de lait de vache a été employé pour en exclure la présence de fibrinogène, en le comparant à la thrombine bovine standard : le résultat de cette épreuve a été négatif, ce qui permet d'exclure la présence de fibrinogène dans le substrat que nous avons employé (ce qui d'ailleurs avait déjà été souligné par d'autres auteurs). Parallèlement on a comparé la même thrombine standard avec un substrat de fibrinogène bovin purifié et standardisé : dans ce cas la coagulation se produisait immédiatement (fig. 3-a). Puisqu'on avait exclu la possibilité de la présence de facteurs de la troisième phase, nous nous sommes orientés vers la deuxième phase, en comparant le même substrat de lait avec de la thromboplastine calcique standard commerciale + fibrinogène. Même dans ce cas le résultat a été négatif.

Il y a lieu de remarquer que la thromboplastine calcique employée était très active, son temps de coagulation par rapport à 


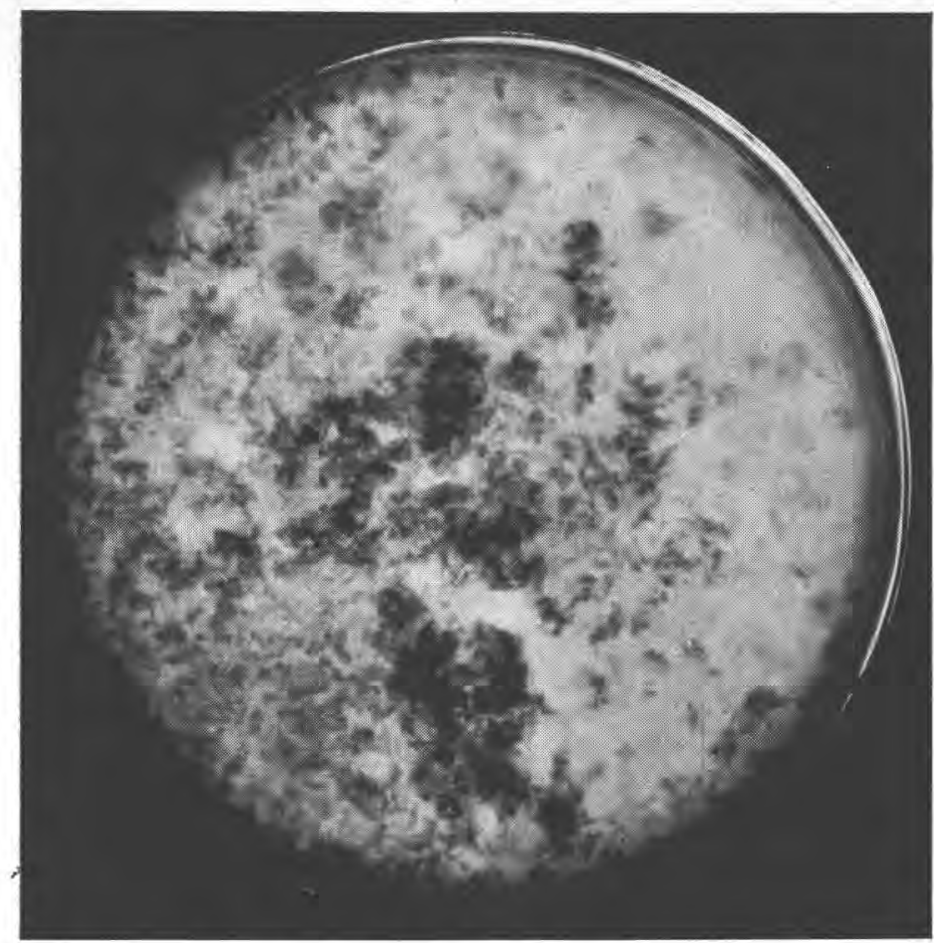

FIG. 1. - Coagulum de lait de vache traité avec $\mathrm{HCl}$

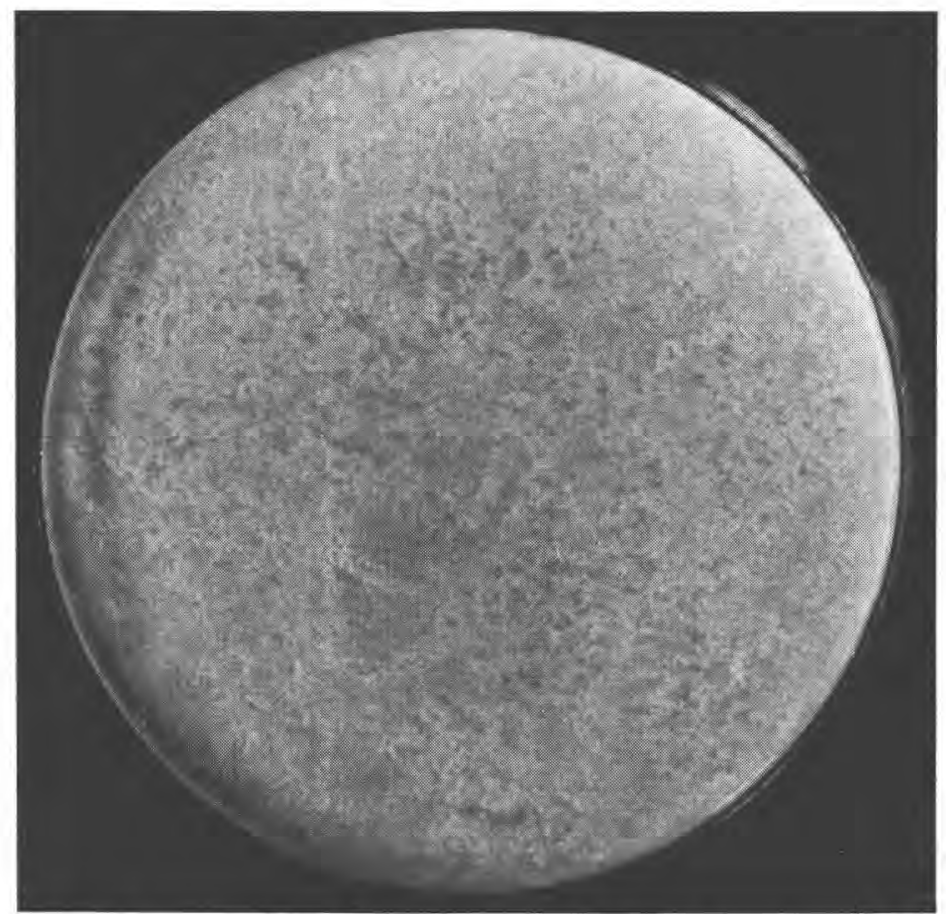

FIG. 2. - Coagulum de lait de vache héparinisé traité avec $\mathrm{HCl}$ (Héparine 5 p. 100 cc. 5 + Lait de vache cc. 100) 
celui du plasma normal étant de $13 \mathrm{~s}$ à 100 p. 100 d'activité (fig. $3-b)$. Cela pouvait être considéré comme une confirmation indirecte de l'inefficacité du dicoumarol, comme déjà dit, aux effets de la modification des épreuves de coagulation du lait que nous avions réalisées.

Il faut ajouter que le substrat de lait de vache que nous avons employé ne contenait ni prothrombine ni facteur V (pro-accélérine) ni facteur VII (proconvertine).

Par exclusion, nous sommes donc arrivés à la première phase de coagulation, qui, en effet, était l'objet de nos recherches.

\section{Méthodes}

$\mathrm{Au}$ début les échantillons choisis pour les recherches de laboratoire étaient :

- du plasma de lait de vache, du lait de vache écrémé,

- du lait de vache entier, du lait humain,

- du sérum de lait de vache avec du $\mathrm{CaCl}_{2}$,

- du lait de vache pasteurisé à $90^{\circ}$ pendant 30 minutes,

- du lait de vache stérilisé.

FIG. 3

a)

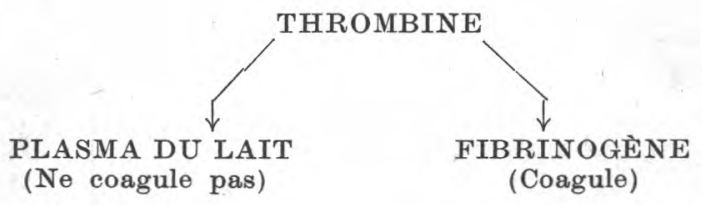

b)

THROMBOPLASTINE

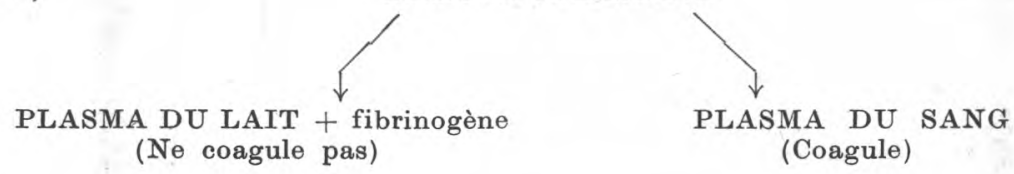

c)

PLASMA DU LAIT

POOL DE PLASMAS HUMAINS

(Coagule) 
Ces substances ont été employées au lieu d'une thromboplastine calcique normale dans le temps de Quick [5]. La température d'incubation des échantillons était de $37^{\circ} \mathrm{C}$. Le substrat de plasma humain était un lyophilisé commercial obtenu d'individus sûrement normaux : il s'agissait done d'un " pool » de plasmas qu'on contrôlait au fur et à mesure avec de la thromboplastine calcique commerciale.

Ensuite pour les épreuves successives on a employé exclusivement du lait de vache entier cru et du lait humain entier cru, car on avait remarqué que la présence d'un pourcentage supérieur ou inférieur de caséine n'influençait pas sensiblement les épreuves d'hémocoagulation.

Pour ce qui concerne la technique de la génération thromboplastinique plasmatique appliquée au lait, nous nous sommes tenus aux dosages classiques de l'hématologie qu'on a reproduits dans les tableaux.

\section{Résultats}

Une série de déterminations du temps de Quick où on avait mélangé à la température de $37^{\circ} \mathrm{C}, 0,4 \mathrm{~cm}^{3}$ de lait avec $0,2 \mathrm{~cm}^{3} \mathrm{de}$ substrat plasmatique humain lyophilisé et ramené à volume, a fourni des résultats très positifs (fig. 3-c).

En employant du lait de vache au lieu de la thromboplastine calcique les temps obtenus allaient de 5 à 6 minutes pour toutes les épreuves (20) faites (tableau I). Il y a lieu de remarquer qu'on n'a pas ajouté, comme dans le temps de Quick, du $\mathrm{CaCl}_{2} 0,025 \mathrm{M}$, qui est un facteur accélérant la coagulation.

Vu ces précédents, même si plutôt génériques, il faut souligner que cette action thromboplastinique du lait s'accordait avec ce que nous avions constaté dans les épreuves de coagulation du lait de vache en ajoutant l'héparine. En effet cette dernière substance exerce une action du type antithromboplastinique.

TABLEAU I

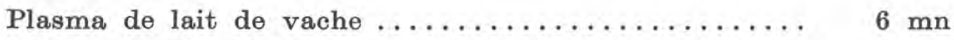

Lait de vache écrémé .................. 4 mn $35 \mathrm{~s}$

Lait de vache entier .................. 4 mn 30 s

Lait humain ...................... 0 mn $30 \mathrm{~s}$

Sérum de lait de vache $+\mathrm{CaCl}_{2} 0,025 / \mathrm{M} \ldots \ldots \ldots \ldots \ldots \mathrm{mn} 25 \mathrm{~s}$

Lait de vache pasteurisé à $90^{\circ}$ pour 30 minutes .... $6 \mathrm{mn} 30 \mathrm{~s}$

Lait de vache stérilisé .................. $11 \mathrm{mn} 45 \mathrm{~s}$

Contrôle (thromboplastine calcique)............. 0 mn $13 \mathrm{~s}$ 


\begin{tabular}{|c|c|}
\hline Epreuves effectuées sur lait de vache & $\begin{array}{l}\text { Moyenne des temps } \\
\text { de coagulation }\end{array}$ \\
\hline 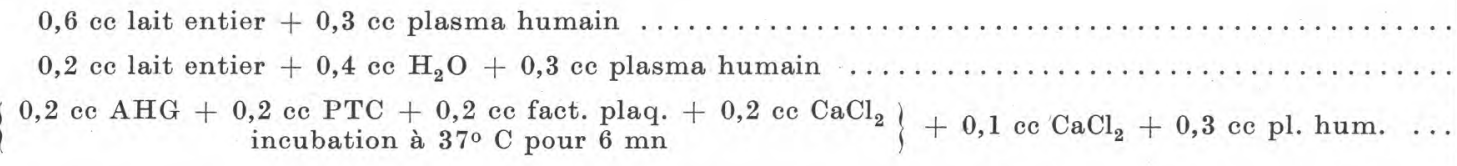 & $\begin{array}{r}5 \mathrm{mn} 50 \mathrm{~s} \\
11 \mathrm{mn} 40 \mathrm{~s} \\
11 \mathrm{~s}\end{array}$ \\
\hline 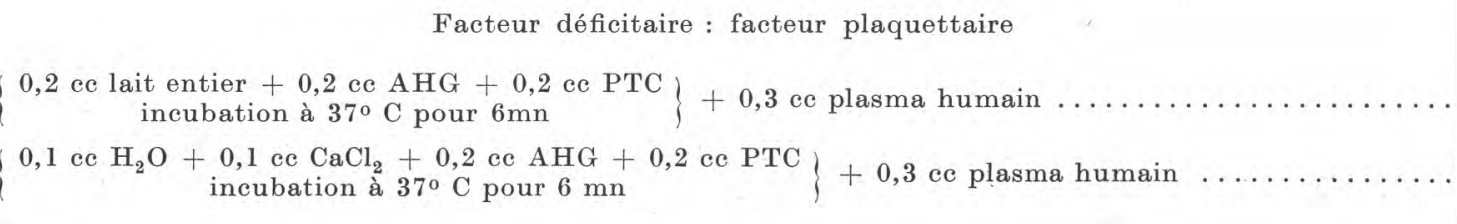 & Pas de coagulation \\
\hline $\begin{array}{c}\text { Facteur déficitaire : PTC } \\
\left\{\begin{array}{c}0,2 \text { cc lait entier }+0,2 \text { ec } \mathrm{AHG}+0,2 \text { ce facteur plaquettaire } \\
\text { incubation à } 37^{\circ} \mathrm{C} \text { pour } 6 \mathrm{mn}\end{array}\right\}+0,3 \text { ce plasma humain } \ldots \ldots \ldots \\
\left.0,1 \text { ec } \mathrm{H}_{2} \mathrm{O}+\begin{array}{c}0,1 \text { ce } \mathrm{CaCl}_{2}+0,2 \mathrm{ec} \mathrm{AHG}+0,2 \text { facteur plaquettaire } \\
\text { incubation à } 37^{\circ} \mathrm{C} \text { pour } 6 \mathrm{mn}\end{array}\right\}+0,3 \text { ce plasma humain } \ldots . .\end{array}$ & $\begin{array}{c}9 \mathrm{mn} 30 \mathrm{~s} \\
\text { Pas de coagulation }\end{array}$ \\
\hline $\begin{array}{c}\text { Facteur déficitaire : AHG } \\
\left\{\begin{array}{c}0,2 \text { cc lait entier }+0,2 \text { ce PTC }+0,2 \text { ce facteur plaquettaire } \\
\text { incubation à } 37^{\circ} \mathrm{C} \text { pour } 6 \mathrm{mn}\end{array}\right\}+0,3 \text { ce plasma humain } \ldots \ldots \ldots \ldots \\
\begin{array}{l}0,1 \text { cc } \mathrm{H}_{2} \mathrm{O}+0,1 \text { cc } \mathrm{CaCl}_{2}+0,2 \text { PTC }+0,2 \text { facteur plaquettaire } \\
\text { incubation à } 37^{\circ} \mathrm{C} \text { pour } 6 \mathrm{mn}\end{array}\end{array}$ & $\begin{array}{l}5 \mathrm{mn} 25 \mathrm{~s} \\
\text { Pas de coagulation }\end{array}$ \\
\hline
\end{tabular}




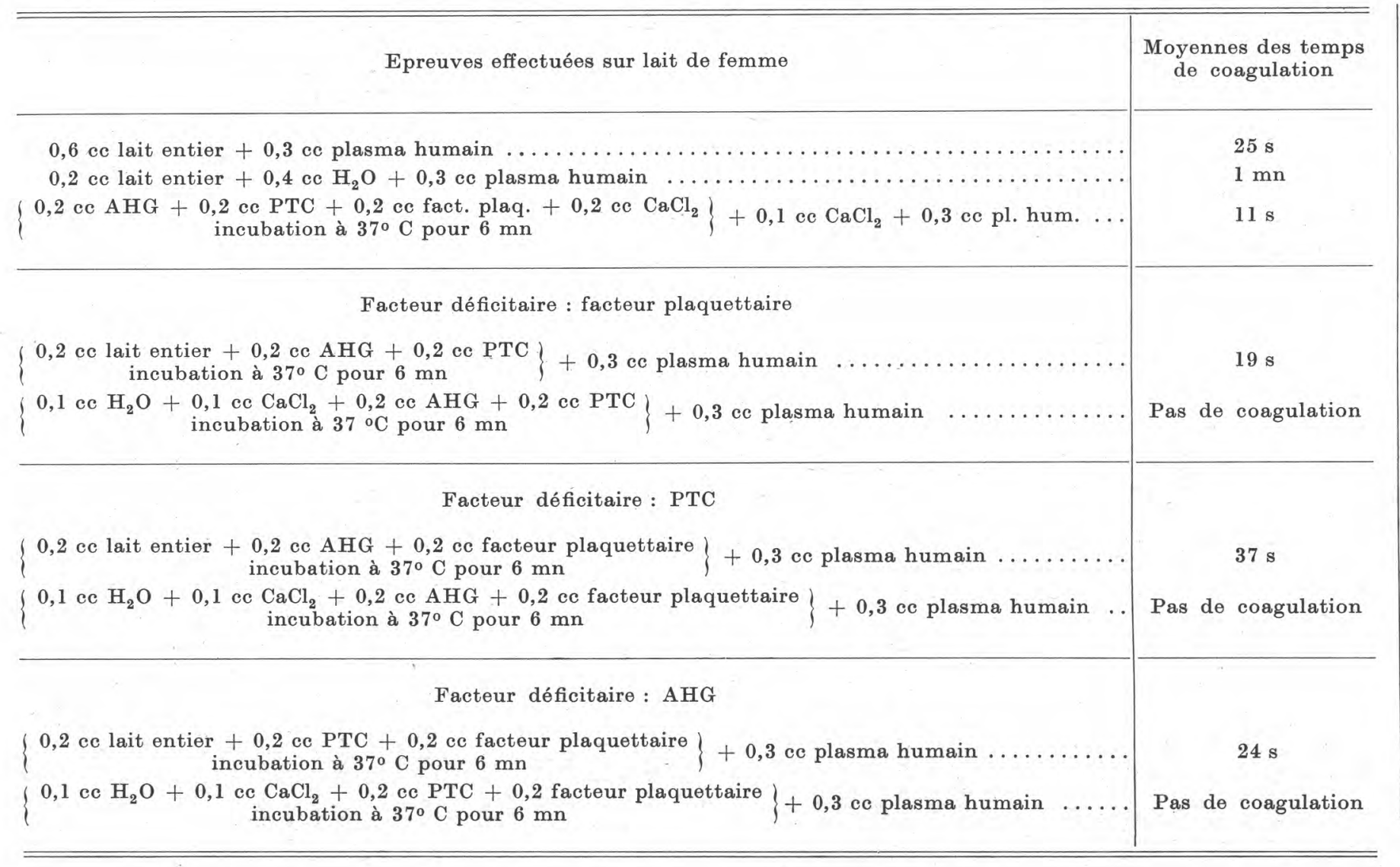


Des valeurs indiquées sur le tableau I, il ressort le haut pouvoir thromboplastinique du lait humain qui s'approche de celui de la thromboplastine calcique. Il faut encore noter que pas toute l'activité thromboplastinique n'est complètement détruite par les températures élevées, comme le démontrent les épreuves avec du lait pasteurisé et stérilisé.

D'après les précédentes expériences, nous avons essayé d'approfondir la recherche en examinant le plasma du lait avec la technique de la génération thromboplastinique plasmatique.

Cette technique exploite les propriétés particulières de plusieurs facteurs de la première phase de coagulation, intéressés par la génération thromboplastinique. En particulier, ce sont trois facteurs qui jouent leur rôle dans la réalisation du test en question, c'est-àdire le facteur plaquettaire, la globuline antihémophilique " $\mathrm{A}$ » (1) et le facteur F-C (facteur Christmas) (2). Normalement, ce mélange engendre de la thromboplastine dans les $6 \mathrm{mn}$ à partir du commencement de la réaction (ou mieux à partir du commencement des réactions entre les trois facteurs susdits plus $\mathrm{CaCl}_{2} 0,025 \mathrm{M}$ ). La thromboplastine qui en résulte, comparée à un substrat de plasma normal standard, donne des temps d'environ $16 \mathrm{~s}$ dans des conditions normales.

Ceci dit, il y a lieu de souligner que dans ce test de génération thromboplastinique nous avons remplacé tour à tour dans le mélange de génération, un des facteurs par le substrat de lait de vache en laissant inaltérés les autres facteurs. Il faut remarquer encore que dans le mélange de génération on n'a pas ajouté du $\mathrm{CaCl}_{2} 0,025 \mathrm{M}$.

Pour ceux qui n'ont pas de familiarité avec les techniques d'hématologie, nous remarquerons qu'en général on soumet 0,2 $\mathrm{cm}^{3}$ de substance en examen (ordinairement le sang, dans notre cas le lait) à incubation pour 6-7 mn, alternativement avec deux des trois facteurs de génération thromboplastinique, respectivement pris en quantité de $0,2 \mathrm{~cm}^{3}$. Ensuite ce mélange, dont le volume total est de $0,6 \mathrm{~cm}^{3}$, est mis en contact avec $0,3 \mathrm{~cm}^{3}$ de substrat de plasma humain.

Il est évident que lorsqu'on mélange $0,2 \mathrm{~cm}^{3}$ de lait à $0,4 \mathrm{~cm}^{3}$ de substances étrangères, ses propriétés en sont affaiblies; c'est pourquoi nous avons voulu, avant tout, contrôler les temps de coagulation en mélangeant $0,2 \mathrm{~cm}^{3}$ de lait avec $0,4 \mathrm{~cm}^{3}$ d'eau distillée afin de pouvoir les comparer avec ceux 'qu'on obtenait en mélangeant le lait avec deux des trois facteurs thromboplastiniques, alternativement.

Les combinaisons possibles sont :

1) lait $0,2 \mathrm{~cm}^{3}+$ facteur globuline antihémophilique $\mathrm{A}+$ facteur Christmas $0,2 \mathrm{~cm}^{3}$ (déficit du facteur plaquettaire)

(1) AHG.

(2) PTG. 
2) facteur plaquettaire $0,2 \mathrm{~cm}^{3}+$ lait $0,2 \mathrm{~cm}^{3}+$ facteur Christmas (déficit du facteur antihémophilique $\mathrm{A}$ )

3) facteur plaquettaire $0,2 \mathrm{~cm}^{3}+$ facteur antihémophilique A $0,2 \mathrm{~cm}^{3}$ + lait $0,2 \mathrm{~cm}^{3}$ (déficit facteur Christmas).

Les résultats de ces épreuves semblent significatifs et particulièrement le fait nous semble intéressant que, malgré les différents types de mélanges employés, le coagulum se présentait toujours avec une avance remarquable par rapport aux épreuves en blanc. Les temps moyens obtenus pour le lait de vache allaient de 6-7 mn quand le facteur plaquettaire était déficitaire à $9 \mathrm{mn} 30 \mathrm{~s}$ quand le facteur Christmas était déficitaire. Le déficit du facteur globuline antihémophilique A avait donné un temps de $5 \mathrm{mn} 25 \mathrm{~s}$.

On fait remarquer que $0,2 \mathrm{~cm}^{3}$ de lait mélangé à $0,4 \mathrm{~cm}^{3}$ d'eau distillée donnaient un temps de $11 \mathrm{mn} 40 \mathrm{~s}$.

Pour ce qui concerne le lait humain, les différents temps obtenus ont été dans l'ordre : 19,37 et $24 \mathrm{~s}$. L'épreuve en blanc avec $\mathrm{H}_{2} \mathrm{O}$ a donné un temps de $60 \mathrm{~s}$.

Après cela, même si les temps étaient différents, les différents types de laits examinés remplaçaient validement dans le mélange de génération chaque facteur déterminant aux effets de la génération thromboplastinique.

Les tableaux no II et III indiquent synthétiquement les valeurs obtenues dans les différentes épreuves comparées, pour plus de clarté et de précision, avec toutes celles des différentes épreuves en blanc.

Nous précisons que les données représentent la moyenne des temps obtenus au cours des expérimentations puisque, naturellement, on a employé des laits différents provenant de différents lots.

D'après ces résultats préliminaires que nous avons obtenus, nous pensons qu'une action thromboplastinique peut être, sans doute aucun, reconnue au lait, tant humain que de vache.

Cette affirmation de notre part se limite, pour le moment, à diffuser des résultats fondamentaux et nous nous promettons dans un de nos travaux ultérieurs de rapporter les résultats des recherches spécifiques que nous allons faire pour démontrer l'action thromboplastinique effective du lait, en en comparant les facteurs éventuels à ceux qui sont bien connus pour la coagulation du sang.

\section{Résumé}

Les auteurs relatent au sujet des effets anticoagulants de l'héparine sur le lait, soit pour ce qui concerne la coagulation par la présure, soit pour ce qui concerne celle chimique avec de l'acide chlorhydrique. 
Après cela ils sont en état de faire ressortir par les techniques normales d'hématologie une activité thromboplastinique considérable soit du lait humain soit du lait de vache.

Ils complètent leur recherche, en appliquant la méthode de la régénération thromboplastinique et ils font remarquer comme tous les deux types de lait peuvent s'insérer dans la réaction, en substituant chaque fois chacun des trois facteurs fondamentaux de la première phase de l'hémocoagulation, que les auteurs avaient tenu alternativement déficitaire dans les mélanges différents en examen.

\section{Summary}

The authors describe the anticoagulant effects of heparin in milk, during renneting and in coagulation with hydrochloric acid.

Using normal hematological techniques, they found that thromboplastin is active both in human and cow's milk.

They establish that in thromboplastin generation, both types of milk can substitute any one of three factors necessary in the first phase of blood clotting.

\section{RÉFÉRENCES BIBLIOGRAPHIQUES}

[1] Buruiana L. - Le Lait, 1941, vol. XXI (138).

[2] Kopaczewski W. - Phisico-Chimie du Lait. Dunod. Paris 1950.

[3] Marcais H. - Le Lait. 1965, vol. XLV (241-250).

[4] Frentz R. et Dreuilhe. - Le Lait. 1965, vol. XLV (489-508).

[5] Raggio-Guarnaschelli-Gigante. - Analisi Cliniche (p. 714). - Il Peensiero Scientifico. Roma. 